\title{
ON THE CONSTRUCTION OF THE BOCKSTEIN SPECTRAL SEQUENCE
}

\author{
BY \\ JERROLD SIEGEL
}

ABSTRACT. The Bockstein spectral sequence is developed from a direct limit construction. This is shown to clarify its relation to certain associated structures, in particular the divided power operations. Finally, the direct limit construction is used to study the problem of enumerating the Bockstein spectral sequences over a given simple $R$-module.

The Bockstein homomorphism can be defined as follows: Let $(M, d)$ be a free chain complex of abelian groups. Let $\left(C^{*}\left(M, Z_{p}\right), d^{*}\right)$ be the associated cochain complex with $Z_{p}$-coefficients and let $x \in H^{n}\left(M, Z_{p}\right)$. Finally let $c \in$ $C^{n}(M, Z)$ be a cochain whose mod $p$ reduction represents $x$. One checks that the above implies $d^{*} c=p \cdot e$ for some cocycle $e \in C^{n+1}(M, Z)$. Define $\beta_{1} x=$ $[e] \in H^{n+1}\left(M, Z_{p}\right)$. Checking that $\beta_{1}^{2}=0$ define $E_{2}^{*}(M)$ to be the homology of the complex $H^{*}\left(M, Z_{p}\right)$ with respect to the differential $\beta_{1}$.

In general $x \in E_{r}^{*}(M)$ can be represented by chains $c \in C^{n}(M, Z)$ with $d^{*} c$ $=p^{r} e$. Define a differential on $E_{r}^{*}(M)$ by $\beta_{r} x=[e]$ and $E_{r+1}^{*}(M)$ to be homology with respect to $\beta_{r}$. The sequence of graded complexes $E_{r}^{*}(M)$ is the Bockstein spectral sequence with respect to $p$.

More efficiently [1] this spectral sequence can be derived from the coefficient sequence $0 \rightarrow Z \stackrel{p}{\rightarrow} Z \rightarrow Z_{p} \rightarrow 0$ by considering the associated short exact sequence of cochain complexes,

$$
0 \rightarrow C^{*}(M, Z) \rightarrow C^{*}(M, Z) \rightarrow C^{*}\left(M, Z_{p}\right) \rightarrow 0
$$

and forming the exact couple

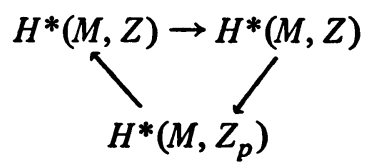

The spectral sequence of this exact couple is the Bockstein spectral sequence.

Received by the editors March 22, 1973 and, in revised form, July 22, 1974.

AMS (MOS) subject classifications (1970). Primary 55J99, 55H99; Secondary 55G05, 55 G20.

Key words and phrases. Bockstein spectral sequences, divided power operations, obstructions, higher order operations, $H$-spaces. 
This definition is the usual starting point of most investigations requiring this spectral sequence. The point we shall try to make below is that for certain formal constructions and structural questions this is not the best definition. Below we offer an alternate formulation of the Bockstein spectral sequence and give applications of this formulation.

In $\S 1$ of this paper we generate the Bockstein spectral sequence by a direct limit construction in a suitable abelian category. The definition above is shown to be related to a dual inverse limit construction. As is often the case, the direct limit construction behaves better. In the second section we offer evidence of this. In particular, we show how the divided power operations [7] may be thought of as operations on the "direct limit" Bockstein exact couple. This leads to certain higher order operations on the Bockstein spectral sequence which appear implicitly in Browder's work on $H$-spaces [1] , [2] , [3]. We show how some of Browder's results appear in our setting.

We next study the classification of Bockstein spectral sequences over a given $R$-module, for example, as short sequences of $Z\left[Z_{2}\right]$-modules. $0 \rightarrow Z \stackrel{2}{\rightarrow} Z \rightarrow Z_{2} \rightarrow 0$ with trivial action, and $0 \rightarrow \hat{Z} \stackrel{2}{\rightarrow} \hat{Z} \rightarrow Z_{2} \rightarrow 0$ (twisted integers) yield different spectral sequences with isomorphic $E_{1}^{*}$-terms. One asks for a classification of all $Z\left[Z_{2}\right]$-Bockstein spectral sequences over $Z_{2}$.

In $\S 3$ we present an obstruction theory which formally gives a classification of the Bockstein spectral sequences over a given $R$-module. This obstruction theory is applied to specific examples including those discussed above.

Finally, we would like to thank the referee for his helpful suggestions on the organization of this paper.

1. The Bockstein spectral sequence in an abelian category. In this section we present an alternative formulation of the Bockstein spectral sequence. We take advantage of the fact that the Bocksteins can also be defined as follows: Let $\delta$ be the connecting homomorphism associated with the coefficient sequence $0 \rightarrow Z_{2} \rightarrow$ $Z_{2^{r+1}} \rightarrow Z_{2^{r}} \rightarrow 0$. Let $\pi: Z_{2^{r}} \rightarrow Z_{2}$ be reduction. Then, also, $\beta_{r}=\delta \pi^{-1}$. Indeed, though we develop the material in this section in the generality of abelian categories for later application, the reader may wish to think of abelian groups and short exact sequences of abelian groups throughout this first section.

1.1. Assumptions. (a) We will assume that we are given an abelian category $A$ and we have a proper class $P$ of short exact sequences in $A[6]$. The groups $\operatorname{Ext}^{n}(A, C)$ below are w.r.t. the class $P$, though we will not mention this explicitly.

(b) We assume that $A$ is a category of coefficients for some cohomology theory $H^{*}(-)$. That is $H^{*}(-)$ is a graded sequence of functors on A (covariant!) and given a s.e.s. in $A$ we get the usual associated long exact sequence with connecting homomorphism of degree +1 . 
At the referee's suggestion we make the following convention: If $f: A \rightarrow A^{\prime}$ we will write $f: H^{*}(A) \rightarrow H^{*}\left(A^{\prime}\right)$ instead of either the contrary notations $f^{*}$ or $f_{*}$. Hopefully this will cause the reader less confusion than the alternatives would.

(c) We assume that $H^{*}$ takes values in an abelian category where direct limits exist and preserve exactness. (No such assumptions are needed for A.)

1.2. Definition. Let $A$ be in A. A Bockstein tower w.r.t. $A$ is an infinitely high commutative diagram of the form

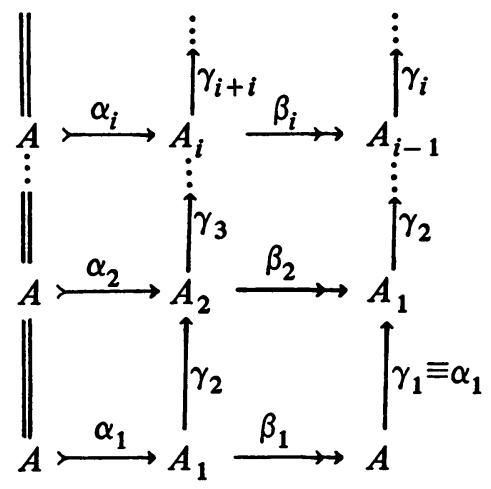

with proper exact rows.

We will write $\left(\alpha_{i} \mid \beta_{i}, \gamma_{i}\right)$ as shorthand for such a diagram.

1.4. Definition. Given a cohomology theory $H^{*}$ (as in 1.1) and a Bockstein tower $\left(\alpha_{i} \mid \beta_{i}, \gamma_{i}\right)$ we define the Bockstein exact couple of $\left(\alpha_{i} \mid \beta_{i}, \gamma_{i}\right)$ with respect to $H^{*}(-)$ to be the graded exact couple

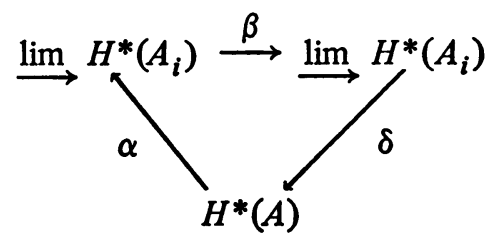

Implicit, among other things, is the observation that the two limits are with respect to the same maps $\gamma_{i}$. We write $H^{*}\left(A_{i}\right)$ for $\lim _{\longrightarrow} H^{*}\left(A_{i}\right)$.

It is the case that the spectral sequence defined by this exact couple does not depend on the maps $\gamma_{i}$ only on the sequences $\alpha_{i} \mid \beta_{i}$. Below we will give a proof of this, but first we give some examples.

1.6. ExAmples. In all examples below $A=A_{0}=Z_{2}$.

(a) Let $A$ be the category of abelian groups $A_{r}=Z_{2}^{r+1}, \alpha_{r}=2^{r}, \gamma_{r}=2$, $\beta_{r}=\pi_{r}: Z_{2^{r}} \rightarrow Z_{2} ;$ then $\left(2^{r} \mid \pi_{r}, 2\right)$ is a tower and, as we shall see, the resulting spectral sequence is isomorphic to the usual one associated with $0 \rightarrow Z \stackrel{2}{\rightarrow} Z \rightarrow$ $Z_{2} \rightarrow 0$.

(b) If $A$ is the category of $Z\left[Z_{2}\right]$-modules, $\alpha_{r}$ and $\beta_{r}$ as above, and $A_{r}=$ $\hat{Z}_{2^{r+1}}$; then the resulting spectral sequence is isomorphic to the one associated 
with $0 \rightarrow \hat{Z} \stackrel{2}{\rightarrow} \hat{Z} \rightarrow Z_{2} \rightarrow 0$. Here the "hat" refers in each instance to the twisting $Z_{2}$-action $(x \rightarrow-x)$.

(c) If $A$ is the category of $Z\left[Z_{4}\right]$-modules.

$$
\begin{aligned}
& A_{0}=Z_{2}, \quad A_{1}=Z_{2} \oplus Z_{2}, \quad A_{2}=Z_{2} \oplus Z_{4}, \\
& A_{2^{r+1}}=Z_{2^{r+1}} \oplus Z_{2^{r+1}}, A_{2 r}=Z_{2^{r}} \oplus Z_{2^{r+1}} .
\end{aligned}
$$

For suitable maps and actions of $Z_{4}$ on $A_{r}$ we get the tower associated with $0 \rightarrow$ $Z[i] \stackrel{i+1}{\longrightarrow} Z[i] \rightarrow Z_{2} \rightarrow 0$. This example was studied in [4].

We first study the nature of the $E_{(r)}$-term in terms of the tower. We set $\beta_{0}$ $=1$ and $\beta^{r}=\beta_{1} \ldots \beta_{r-1} \beta_{r}$.

1.7. LemMa. $\gamma_{r}$ is mono and $\beta^{r}$ is epi and $\gamma_{r} \mid \beta^{r}$.

Proof. $\beta^{r}$ is a composition of epimorphisms and hence an epimorphism; $\gamma_{1}=\alpha_{1}$ is mono and $\gamma_{1} \mid\left(\beta_{1}=\beta^{1}\right)$. Now consider

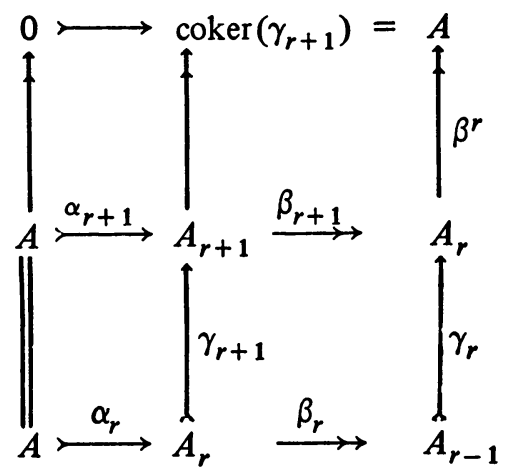

which gives the induction step. $\gamma_{r+1}$ is a mono by the categorical five lemma, $\operatorname{coker}\left(\gamma_{i}\right)=A$ by the $3 \times 3$ lemma [6]. Finally, $\beta^{r+1}=\beta^{r} \beta_{r+1}$.

1.9. LemMA. Let $\gamma_{s}^{r+s}=\gamma_{r+s} \ldots \gamma_{s+1}: A_{s} \rightarrow A_{r+s}$ and $\beta_{s}^{r+s}=\beta_{s} \ldots$ $\beta_{r+s}: A_{r+s} \rightarrow A_{s-1}$. Then we have the following commutative diagram with exact rows:

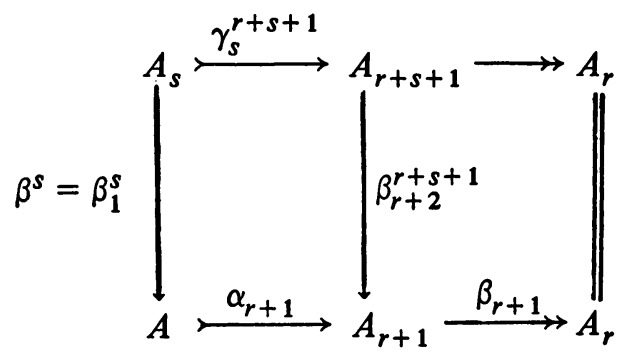


Proof. This lemma also follows from (1.8) by an appropriate alteration of the bottom two squares.

1.10. LEMma. Let $\delta^{r} H^{*}\left(A_{r}\right) \rightarrow H^{*+1}(A)$ be the boundary homomorphism associated with the sequence $A \mapsto A_{r+1} \rightarrow A_{r}$. Then

(a) $\delta^{r} \gamma_{r}=\delta^{r-1}$,

(b) $\operatorname{Im} \delta^{r} \subseteq \operatorname{Im} \beta^{s}$ for $r \geqslant 0, s \geqslant 1$.

Proof. (a) Follows from the bottom two lines of (1.8).

(b) Follows from 1.9.

1.11. LEMMA. $\beta^{r}\left(\delta^{r}\right)^{-1}\left(\delta^{r-1} H^{*}\left(A_{r-1}\right)\right)=\beta^{r+1}\left(H^{*}\left(A_{r+1}\right)\right)$.

Proof.

$$
\begin{aligned}
\beta^{r}\left(\left(\delta^{r}\right)^{-1}\left(\delta^{r-1} H^{*}\left(A_{r-1}\right)\right)\right) & =\beta^{r}\left(\left(\delta^{r}\right)^{-1}\left(\delta^{r} \gamma_{r} H^{*}\left(A_{r-1}\right)\right)\right) \text { by } 1.10(\mathrm{a}) \\
& =\beta^{r}\left(\left(\operatorname{ker} \delta^{r}+\gamma_{r} H^{*}\left(A_{r-1}\right)\right)\right) \\
& =\beta^{r}\left(\operatorname{ker} \delta^{r}\right) \quad \text { since } \beta^{r} \gamma_{r}=0 \text { by } 1.7 .
\end{aligned}
$$
inclusion.

1.12. THEOREM. In the B.S.S. $\operatorname{Im} \beta^{r} / \operatorname{Im} \delta^{r-1} \cong E_{(r+1)}$, the map given by

Proof. Remembering that the $(r+1)$ st differential $\delta_{(r+1)}$ is induced by $\delta(\beta)^{-r} \alpha$ [5] we prove the theorem by induction, setting $\beta^{0}=1$ and $\delta^{-1}=0$.

By induction

and

$$
\begin{aligned}
\operatorname{ker} \delta_{(r+1)} & =\beta^{r}\left(\delta^{r}\right)^{-1}\left(\delta^{r-1}\left(H^{*}\left(A_{r-1}\right)\right)\right) \\
& =\operatorname{Im} \beta^{r+1} \quad \text { by } 1.11,
\end{aligned}
$$

$$
\begin{aligned}
\operatorname{Im} \delta_{(r+1)} & =\operatorname{Im}\left[\delta(\beta)^{-r} \alpha\right]=\operatorname{Im}\left(\delta^{r}\left(\left(\beta^{r}\right)^{-1}\right)\right) \\
& =\operatorname{Im}\left(\delta^{r}\right) .
\end{aligned}
$$

1.12 gives a description of $E_{(r+1)}$ independent of the maps $\gamma_{i}$. A suitable extension of 1.12 shows the $E_{(\infty)}$ is also independent; however this will follow from a simpler argument under mild additional assumptions. First, though, we must relate the usual construction of the Bockstein spectral sequence to the one presented above.

1.13. Definition. A short presentation of an object $A$ is an exact sequence of the form $R \stackrel{\sigma}{\rightarrow} R \stackrel{\rho}{\rightarrow} A$ where $R$ is some object of $A$.

1.14. Construction. Given a short presentation $R \stackrel{\rho}{\rightarrow} R \stackrel{\rho}{\rightarrow} A$ we can associate a Bockstein tower as follows:

Consider the diagram: 


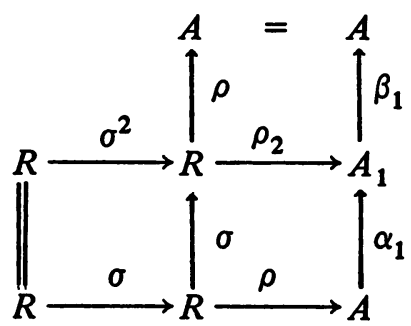

where $A_{1}=\operatorname{coker} \sigma^{2}$ and where $\sigma^{2}=\sigma \circ \sigma$.

We now proceed by induction. Define

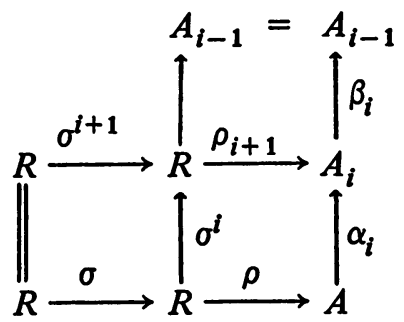

In order to complete the construction we define $\gamma_{i}$ by

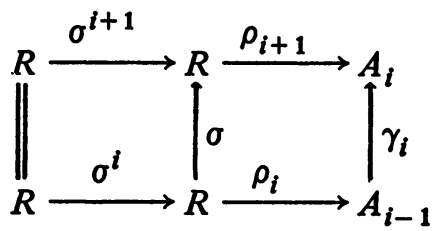

We must only check that

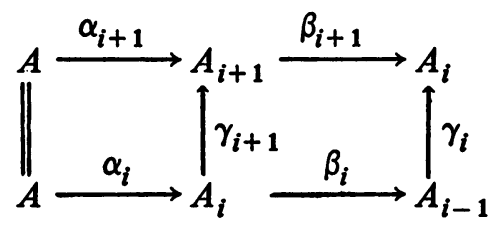

commutes, but this follows since everything in sight is induced by powers of $\sigma$. As an example of this construction one can check that $Z \stackrel{2}{\rightarrow} Z \rightarrow Z_{2}$ induces $\left(2^{i} \mid \pi^{i}, 2\right)$.

1.15. Definition. Given a short presentation $R \stackrel{\sigma}{\rightarrow} R \stackrel{\rho}{\rightarrow} A$ we associate an exact couple

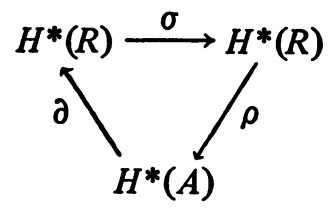


We now show that the spectral sequence associated with this exact couple is isomorphic with the one associated with the associated tower. First we need a definition.

1.16. Definition. (a) Let $I$ be the category whose objects are nonnegative integers $\{0,1, \ldots\}$ and

$$
I(n, m)= \begin{cases}\{\longrightarrow\}, & n \leqslant m, \\ \varnothing, & m<n,\end{cases}
$$

(i.e., a single arrow $n \leqslant m)$. A is included in $A^{I}$, the functor category, by $A(n)$ $=A, A(n \leqslant m)=\mathrm{Id}$.

(b) Let $\hat{R}$ and $\hat{A} \subseteq A^{I}$ be defined as follows:

$$
\begin{aligned}
& \hat{R}(n)=R, \quad \hat{R}(n \leqslant m)=\sigma^{m-n}=\sigma \circ \sigma \circ \ldots, \\
& \hat{A}(n)=A_{n}, \quad \hat{A}(n \leqslant m)=\gamma_{m} \circ \gamma_{m-1} \circ \ldots \circ \gamma_{n+1} .
\end{aligned}
$$

In $A^{I}$ we have the short exact sequence

$$
R \stackrel{\hat{\sigma}}{\longrightarrow} \hat{R} \stackrel{\hat{\rho}}{\longrightarrow} \hat{A}
$$

where $\hat{\sigma}(n): R(n)=R \stackrel{\sigma^{n+1}}{\longrightarrow} R=\hat{R}(n)$ and $\hat{\rho}(n)=\rho_{n}$.

(c) Writing $H^{*}(\hat{B})$ for $\lim _{\longrightarrow} H^{*}(B(n)), \hat{B}$ in $A^{I}$, we have the exact triangle

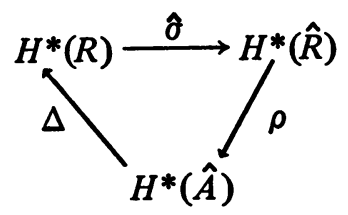

associated with (1.17).

1.18. Theorem. Given a short presentation $R \stackrel{\sigma}{\rightarrow} R \stackrel{\rho}{\rightarrow} A$, let $\left(\alpha_{i} \mid \beta_{i}, \gamma_{i}\right)$ be the associated Bockstein tower. The spectral sequence associated with ol $\rho$ is isomorphic with the spectral sequence associated with $\left(\alpha_{i} \mid \beta_{i}, \gamma_{i}\right)$. In particular the following diagram commutes:

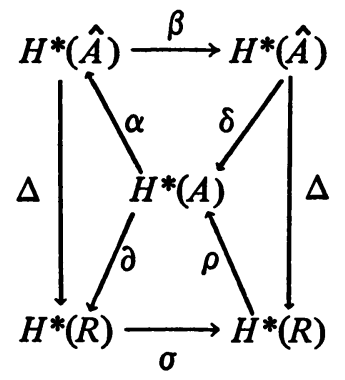

(Note: $\stackrel{H}{\rightarrow}^{*}\left(A_{i}\right) \equiv H^{*}(\hat{A})$.) 
PROOF. The proof consists of proliferating the notation and writing down the appropriate commutative diagram in $A^{I}$.

Let $\hat{A}^{\prime}$ be defined by $\hat{A}^{\prime}(n)=A_{n-1}\left(\hat{A}^{\prime}(0)=0\right), \hat{A}^{\prime}(n \leqslant m)=$ $\hat{A}(n-1 \leqslant m-1)$. Let $\hat{\beta}: \hat{A} \rightarrow \hat{A}^{\prime}$ be defined by $\hat{\beta}(n)=\beta_{n}$. Of course

(a) $(\hat{\beta})=\beta$,

(b) $H^{*}(\hat{A})=H^{*}\left(\hat{A}^{\prime}\right)$.

Let $\hat{\sigma}^{\prime}: R \rightarrow \hat{R}$ be defined by $\hat{\sigma}^{\prime}(n)=\sigma^{n}$. Let $\hat{\rho}^{\prime}: R \rightarrow \hat{A}^{\prime}$ be defined by $\hat{\rho}^{\prime}(n)=\rho_{n-1}$. Again

(c) $\left(\hat{\sigma}^{\prime}\right)=(\hat{\sigma})$,

(d) $\left(\hat{\rho}^{\prime}\right)=(\hat{\rho})$.

We have the following commutative diagram in $A^{I}$ with exact rows:

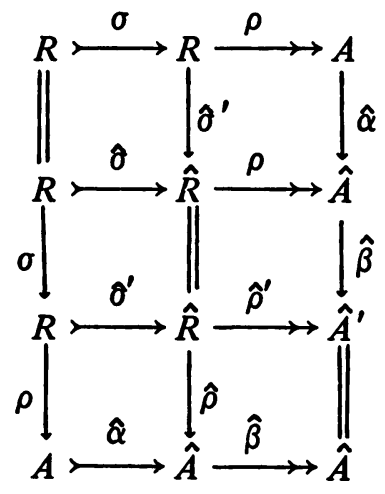

Using the top two rows we have $\Delta \alpha=\partial$. Using the middle two rows and (a) above we have $\sigma \Delta=\beta \Delta$. Using the bottom two rows we have $\rho \Delta=\delta$.

1.21. Example. Consider $0 \rightarrow Z \stackrel{2}{\rightarrow} Z \stackrel{\pi}{\rightarrow} Z_{2} \rightarrow 0$. Taking limits in the category of abelian groups we have $\lim _{\longrightarrow} Z=Z\left[\frac{1}{2}\right]$, rationals of form $p / 2$.

$$
\stackrel{\lim }{\longrightarrow} Z_{2^{k}} \cong C_{2}\left\{e \frac{i \pi p}{2^{k}} \mid p, k \text { integers }\right\}
$$

in the limit (1.20) is

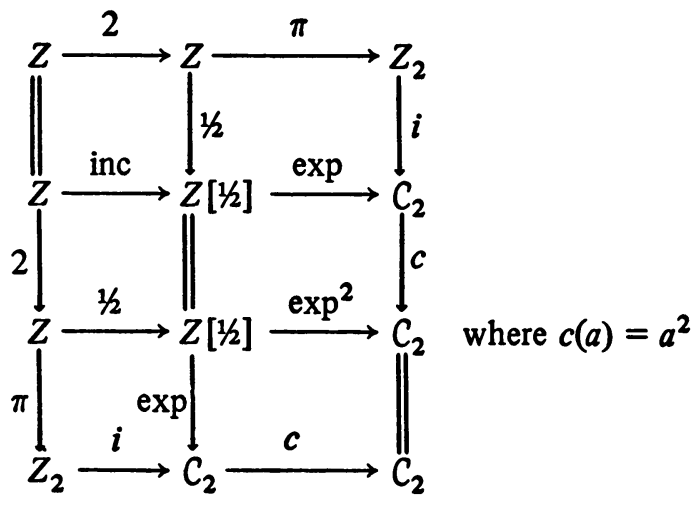


This example suggests a final observation which is used in $\S 2$.

1.22. TheOREM. Let A admit exactness preserving direct limits; then there is a map of exact couples

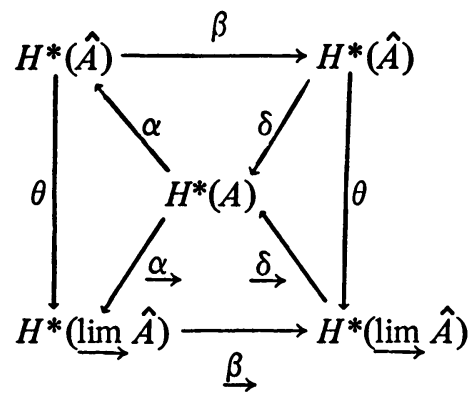

Proof. $\theta$ is induced by the standard maps of the factors of a diagram into the direct limit.

The last question we consider in this section is when can we say a Bockstein tower arises out of a short presentation. The answer is, modulo an assumption, that this is always the case.

1.23. Assumption. $A$ admits inverse limits. Moreover, suppose we are given a contravariant functor $A: I \rightarrow A$ with $A(n \leqslant m)$ an epimorphism. Then $\lim _{\longleftarrow} A \rightarrow A(n)$ is an epimorphism for all $n$. (Note this holds in categories where epimorphisms coincide with onto set maps.)

1.24. Lemma. Consider the diagram

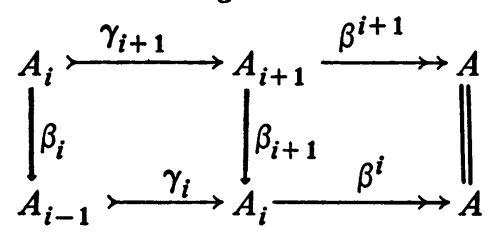

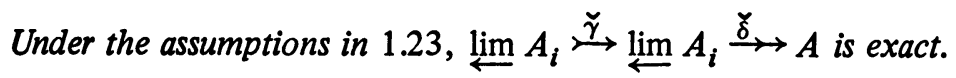

PROof. That $\check{\delta}$ is onto is 1.23 , the rest is categorical.

The proofs of the following two theorems are straightforward, as are their obvious corollaries about the B.S.S.

1.25. THEOREM. Given a Bockstein tower $\left(\alpha_{i} \mid \beta_{i}, \gamma_{i}\right)$, by 1.24 we have the short presentation $\lim A_{i} \stackrel{\gamma}{\longrightarrow} \underset{\lim }{\longleftarrow} A_{i} \stackrel{\delta_{\rightarrow}}{\rightarrow} A$. Then the tower associated with this presentation is isomorphic to $\left(\alpha_{i} \mid \beta_{i}, \gamma_{i}\right)$.

1.26. Theorem. Given a short presentation $R \stackrel{\sigma}{\rightarrow} R \stackrel{\rho}{\rightarrow} A$, let $\left(\alpha_{i} \mid \beta_{i}, \gamma_{i}\right)$ be the associated tower. Then the map $\check{\rho}=\lim \rho_{i}$ gives the commutative diagram 


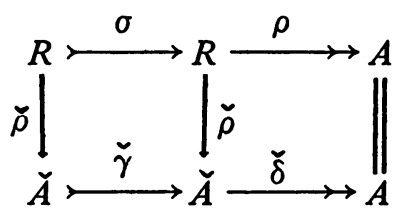

1.27. THEOREM. Let $\left(\alpha_{i} \mid \beta_{i}, \gamma_{i}\right)$ and $\left(\alpha_{i} \mid \beta_{i}, \gamma_{i}^{\prime}\right)$ be two towers (same sequence of extensions). Then $\hat{\delta}=\hat{\delta}^{\prime}$. Hence there is a morphism $\tau$ making the following diagram commute.

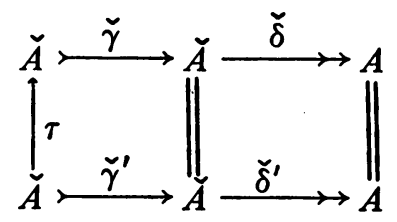

Proof. Since $\check{A}$ is defined only in terms of $\beta_{i}$, the diagram makes sense. But $\check{\delta}=\check{\delta}^{\prime}=\lim \beta^{i}$; hence $\check{\gamma}$ and $\check{\gamma}^{\prime}$ are both kernels of the same map.

1.28. COROLlaRY. The spectral sequence of a tower is independent of the maps $\gamma_{i}$.

2. An application. In this section we extend the divided power operations [7] to operations in the direct limit Bockstein exact couple. We use these operations to define secondary cohomology operations on the Bockstein exact couple. Finally, we use the secondary operation to define a pairing in the Bockstein spectral sequence. The properties of this pairing lead immediately to Browder's 1 -implication lemmas for $H$-spaces [1].

We next consider the construction of the dual pairing. In the case of homotopy-commutative $H$-spaces it is possible, but in the case of homology-commutative $H$-spaces it is only possible for $E^{(r)}, r>1$. The difference between these two cases is reflected in [2] and [3]. We work with $Z_{2}$ and the Pontrjagin square for simplicity.

2.1. The Pontruagin SQUare [7]. There is a cohomology operation

$$
P_{2}: H^{2 m}\left(X, Z_{2}\right) \rightarrow H^{4 m}\left(X, Z_{4}\right)
$$

such that

(a) $P_{2}(x+y)=P_{2}(x)+P_{2}(y)+2 *(x \cdot y)$, where $x \cdot y$ is ordinary cup product.

(b) If $\pi: Z_{4} \rightarrow Z_{2}$ and $x \in H^{2 m}\left(X, Z_{2}\right)$; then $\pi\left(P_{2}(x)\right)=x^{2}$.

(c) If $x^{\prime} \in H^{2 m}\left(X, Z_{4}\right)$; then $P_{2}\left(\pi\left(x^{\prime}\right)\right)=\left(x^{\prime}\right)^{2}$.

Recall from 1.22 that we have the short exact sequence $0 \rightarrow Z_{2} \stackrel{i}{\rightarrow} C_{2} \stackrel{c}{\rightarrow} C_{2} \rightarrow 0$. 


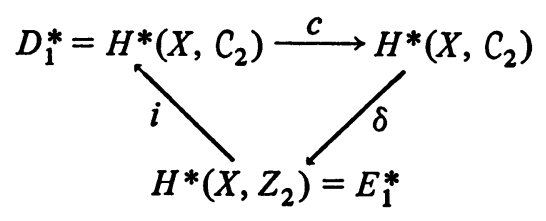

is isomorphic to the spectral sequence associated with the s.e.s. $0 \rightarrow Z \stackrel{2}{\rightarrow} Z \rightarrow$ $Z_{2} \rightarrow 0$.

Proof (1.22). The map $\theta(X)$ of 1.22 is, of course, just the limit of $\theta_{k}(X): H^{*}\left(X, Z_{2 k}\right) \rightarrow H^{*}\left(X, C_{2}\right)$ induced by the inclusion. We will write $\hat{x}$ for $\theta_{k}(X)(x), x \in H^{*}\left(X, Z_{2}\right)$ and leave the appropriate " $k$ " implicit.

2.3. Definition. We define a cohomology operation $\hat{P}_{2}: H^{2 m}\left(X, Z_{2}\right) \rightarrow$ $H^{4 m}\left(X, C_{2}\right)$ by $\hat{P}_{2}(x)=\widehat{\left.P_{2}(x)\right)}$. Note this can be thought of as an operation $\hat{P}_{2}^{1}: E_{(1)}^{2 m} \rightarrow D_{(1)}^{4 m}$.

2.4. THEOREM. $\hat{P}_{2}^{1}$ induces operations $\hat{P}_{2}^{r}: E_{(r)}^{2 m} \rightarrow D_{(r)}^{4 m}$ such that

(a) $\hat{P}_{2}^{r}(x+y)=\hat{P}_{2}^{r}(x)+\hat{P}_{2}^{r}(y)+\widehat{(x \cdot y)}$,

(b) $c \hat{P}_{2}^{r}(x)=\widehat{x^{2}}$.

PROof. First recall from 1.12 that if $x \in E_{r}^{n}$ is . $\ni . \delta_{(r)}(x)=0$ then there exists $z^{\prime} \in H^{n}\left(X, Z_{2^{r+1}}\right) . \ni . \pi z^{\prime}=z$, where $x=[z]$ and $\pi: Z_{2^{r+1}} \rightarrow Z_{2}$.

The theorem now follows by induction on $r$; for $r=1$ the theorem is just the definitions 2.3 and 2.1 .

Now assume the theorem for $r$. By the above $\delta_{(r)} x=0$ implies that there exists a $z^{\prime} \in H^{n}\left(X, Z_{2^{r+1}}\right)$ with $x=\left[\pi^{(r+1)} z^{\prime}\right]$. But by $2.1(\mathrm{c})$

$$
P_{2}(z)=\left(\pi^{r} z^{\prime}\right)^{2} \text { so } P_{2}^{r} x=\widehat{\left(\pi^{r} z^{\prime}\right)^{2}}=(c)^{r\left(z^{\prime}\right)^{2}} \in D_{r+1}^{4 m} \text {. }
$$

In order to finish the proof we must show $\hat{P}_{2}^{r}\left(x+\delta_{(r)} y\right)=\hat{P}_{2}^{r}(x)$ when $\delta_{(r)} x=0$.

By induction

$$
\hat{P}_{2}^{r}\left(x+\delta_{r} y\right)=\hat{P}_{2}^{r}(x)+\hat{P}_{2}^{r}\left(\delta_{(r)} y\right)+\widehat{x \cdot \delta_{(r)}(y)}
$$

but

$$
\widehat{x \cdot \delta_{(r)}(y)}=\widehat{\delta_{(r)}(x \cdot y)}=i \delta_{(r)}(x \cdot y)=0
$$

(this last equality by decomposing $\delta_{(r)}$ in the $r$ th derived couple).

We will be done when we show $\hat{P}_{2}^{r}\left(\delta_{(r)} y\right)=0$. But since $\delta_{(r)}$ is induced by $\delta\left(c^{r-1}\right)^{-1} i$ it clearly suffices to show 
LEMma. $\widehat{P_{2}\left(\delta y^{\prime}\right)}=0$ for $y^{\prime} \in H^{2 m}\left(X, C_{2}\right)$.

Proof. Consider the coefficient diagram

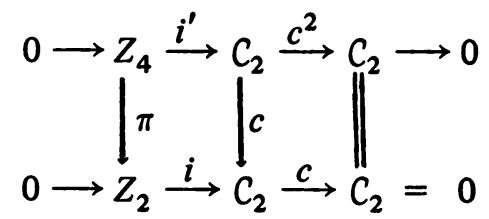

Let $\delta^{\prime}$ be the boundary homomorphism with respect to the top row.

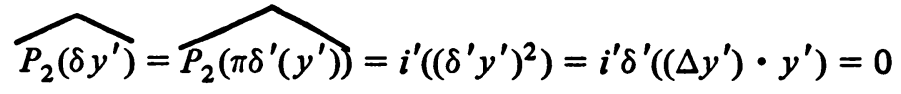

where $\Delta$ is as in 1.16 and products are taken with respect to the pairing $Z \otimes C_{2}$ $\rightarrow \mathrm{C}_{2}$.

Finally, we observe that the formulas (a) and (b) are induced by the corresponding formulas on $P_{\mathbf{2}}$.

We now define a "secondary" operation that appears implicitly in the work of Browder [1].

2.5. Definition. Let $q_{r}^{2 m}: E_{(r)}^{2 m} \rightarrow D_{(r)}^{4 m}$ be defined by $q_{r}^{2 m}(x)=i\left(x^{2}\right)$ $=\widehat{x^{2}}$. We define $\Phi_{r}: \operatorname{ker} q_{r} \rightarrow E_{(r)}^{4 m} / \operatorname{Im}\left(\delta_{r}^{4 m-1}\right)$ by $\Phi_{r}(x)=\left[i^{-1} \hat{P}_{2}^{r}(x)\right]$. Note: $\delta_{r}^{4 m-1}$ is the boundary homomorphism in the $r$ th derived couple not $\delta_{(r)}^{4 m-1}$.

We are now in a position to define the pairing in which we shall be interested.

Let $\left(E_{n}^{(r)}, \partial_{r}\right)$ be the homology B.S.S. Let $\langle$,$\rangle denote the usual pairing$ $E_{n}^{(r)} \otimes E_{(r)}^{n} \rightarrow Z_{2}[1]$.

2.6. Definition. Let $x \in \operatorname{Im} \partial_{(r)}^{4 m} \in E_{4 m}^{r}$ and $y \in \operatorname{ker} q_{r}^{2 m}$. Define $\varphi(x, y)=\left\langle x, \Phi_{r}(y)\right\rangle$.

2.7. THEOREM. $\varphi$ : $\operatorname{Im} \partial_{(r)}^{4 m} \times \operatorname{ker} q_{r}^{2 m} \rightarrow Z_{2}$ is a well-defined pairing $\ni$.

(a) $\varphi\left(x_{1}+x_{2}, y\right)=\varphi\left(x_{1}, y\right)+\varphi\left(x_{2}, y\right)$.

(b) $\varphi\left(x, y_{1}+y_{2}\right)=\varphi\left(x, y_{1}\right)+\varphi\left(x, y_{2}\right)+\left\langle x, y_{1} y_{2}\right\rangle$.

(c) If $f: X \rightarrow X^{\prime}$ then $\varphi\left(f_{*}(x), y^{\prime}\right)=\varphi\left(x, f^{*}\left(y^{\prime}\right)\right)$.

Proof. Since $y \in \operatorname{ker} q_{r}^{2 m}, \varphi(x, y)$ is defined and is unique up to elements of the form $\left\langle x, \delta_{r}^{4 m-1} y^{\prime}\right\rangle$ but $x=\partial_{(r)}^{4 m} x^{\prime}$ so

$$
\begin{aligned}
\left\langle x, \delta_{r}^{4 m-1} y^{\prime}\right\rangle & =\left\langle\partial_{(r)}^{4 m} x^{\prime}, \delta_{r}^{4 m-1} y^{\prime}\right\rangle=\left\langle x^{\prime}, \delta_{(r)}^{4 m}\left(\delta_{r}^{4 m-1} y^{\prime}\right)\right\rangle \\
& =\left\langle x^{\prime}, \delta_{r}^{4 m} i^{*} \delta_{r}^{4 m-1} y^{\prime}\right\rangle=\left\langle x^{\prime}, \delta_{r}^{4 m}(0)\right\rangle=0 .
\end{aligned}
$$

Again, the formulas follow from the corresponding formulas on $\langle$,$\rangle and$ $\hat{P}_{2}^{r}$.

We now give some applications of the pairing. We assume $X$ is an $H$-space with $\mu: X \times X \rightarrow X$ the multiplication and $\left(\pi_{2}, \pi_{2}\right),\left(i_{1}, i_{2}\right)$ the projections and inclusions along the factors. 
2.8. THEOREM. Let $X$ be an H-space, let $0 \neq \bar{x} \in \operatorname{ker} q_{r}^{2 m}$ be primitive. Let $x$ be its dual. Assume $x \in \operatorname{Im} \partial_{(r)}^{4 m}$; then $\varphi\left(x^{2}, \bar{x}\right) \neq 0$, hence $x^{2} \neq 0$.

ProOF.

$$
\begin{aligned}
\varphi\left(x^{2}, \bar{x}\right) & =\varphi\left(\mu_{*}(x \otimes x), \bar{x}\right)=\varphi\left(x \otimes x, \mu^{*} \bar{x}\right) \\
& =\varphi(x \otimes x, 1 \otimes \bar{x}+\bar{x} \otimes 1) \\
& =\varphi(x \otimes x, 1 \otimes \bar{x})+\varphi(x \otimes x, \bar{x} \otimes 1)+\langle x \otimes x, \bar{x} \otimes \bar{x}\rangle
\end{aligned}
$$

but

$$
\varphi(x \otimes x, 1 \otimes \bar{x})=\varphi\left(x \otimes x, \pi_{2}^{*}(\bar{x})\right)=\varphi\left(\pi_{*}(x \otimes x), \bar{x}\right)=\varphi(0, \bar{x})=0 .
$$

Similarly $\varphi(x \otimes x, \bar{x} \otimes 1)=0$ so $\varphi\left(x^{2}, \bar{x}\right)=\langle x \otimes x, \bar{x} \otimes \bar{x}\rangle=1 \neq 0$.

Actually, 2.8 follows from a stronger 1-implication lemma and some observations about Hopf algebras. We prove this stronger result modulo lemma which describes the behavior of $\varphi$ with respect to certain products.

Note that no assumption is made about $X$ being an $H$-space.

2.9. Lemma. Let $x \in \operatorname{Im} \partial_{(r)}^{2 m}$ be primitive. Let $y=\Sigma y_{i}^{\prime} \otimes y_{j}^{\prime \prime}, y \in$ ker $q_{r}^{2 m}$, in the B.S.S. of $X \times X$ be .Э. $0<\operatorname{dim} y_{i}^{\prime}<2 m$. Then $\varphi(x \otimes x, y)=0$.

Proof. We do not give full details. One expands $\varphi(x \otimes x, y)$ and uses formulas [7] describing the behavior of $P_{2}$ under products to conclude that all the terms vanish because of dimensional reasons or because one is pairing a primitive homology and a decomposable cohomology class.

2.10. THEOREM. Let $x$ be an H-space. Let $0 \neq x \in \operatorname{Im} \partial_{r}^{2 m}$ be primitive. Let $\bar{x}$ be the dual of $x$ and let $\bar{x} \in \operatorname{ker} q_{r}^{2 m}$. Then $\varphi\left(x^{2}, \bar{x}\right) \neq 0$; hence $x^{2} \neq 0$.

ProOF.

$$
\begin{aligned}
\varphi\left(x^{2}, \bar{x}\right)= & \varphi\left(\mu_{*}(x \otimes x, \bar{x})\right)=\varphi\left(x \otimes x, \mu^{*} \bar{x}\right) \\
= & \varphi(x \otimes x, 1 \otimes \bar{x}+\bar{x} \otimes 1+y), \quad y \text { as in } 2.9, \\
= & \varphi(x \otimes x, 1 \otimes \bar{x}+\bar{x} \otimes 1)+\varphi(x \otimes x, y) \\
& +\langle x \otimes x, y \cdot(1 \otimes \bar{x}+\bar{x} \otimes 1)\rangle,
\end{aligned}
$$

but $\varphi(x \otimes x, y)=0$ by 2.9 , and $\langle x \otimes x, y \cdot(1 \otimes \bar{x}+\bar{x} \otimes 1)\rangle=0$ for dimensional reasons and $\varphi\left(x^{2}, \bar{x}\right)=\varphi(x \otimes x, 1 \otimes \bar{x}+\bar{x} \otimes 1)=1$ as in 2.8 .

The following theorem is an application of the additional strength of 2.10 .

2.11. THEOREM. Let $X$ be an H-space. Let $0 \neq x \in \operatorname{Im} \partial_{(r)}^{2 m}$ be primitive. Suppose $H^{*}(X, Z)$ has no free subgroup in dimensions $2^{k} m$, and suppose $\left(H^{*}(X, Z)\right)_{2}$ (2-torsion subgroup) consists of only elements of order 2 in dimensions $\left(2^{k} m\right)+1$. Then $x^{n} \neq 0$ for all $n$. 
Proof. Since, $x \in \operatorname{Im} \partial_{(r)}^{2 m}$ and primitive and $x^{2} \neq 0$ imply $x^{2} \in \operatorname{Im} \partial_{(r)}^{4 m}$ and primitive, it suffices to show $q^{2 m}(\bar{x})=i\left(\bar{x}^{2}\right)=0$ at each stage of the induction.

We show $i\left(y^{2}\right)=0, y \in H^{2^{k}} m\left(X, Z_{2}\right)$. Consider the following extended version of 1.18 .

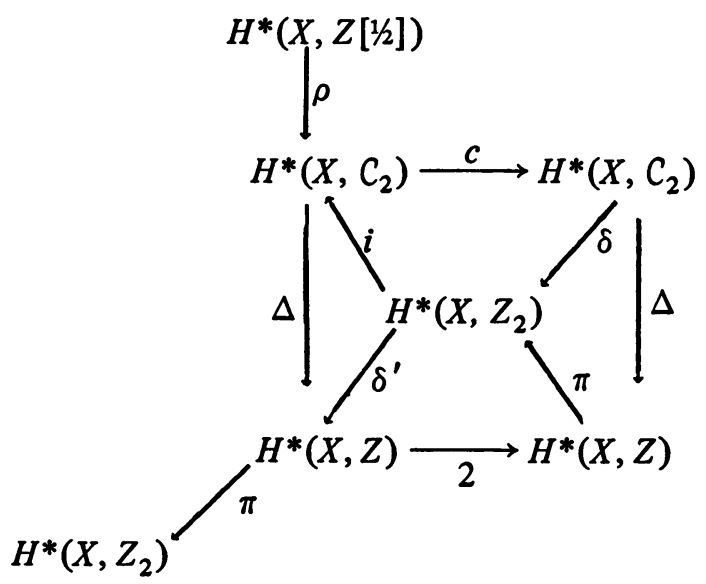

By 1.20-1.21 the right vertical column is exact. But $H^{2{ }^{k}} m(X, Z)$ has no free subgroups so $\left(H^{2^{k} m}(X, Z[1 / 2])\right)_{2}=0$. On the other hand $\left(H^{2^{k}} m\left(X, C_{2}\right)\right)_{2}=$ $H^{2^{k} m}\left(X, C_{2}\right)$ so $\rho=0: H^{2^{k}} m(X, Z[1 / 2]) \rightarrow H^{2^{k} m}\left(X, C_{2}\right)$. Hence $\Delta$ is a monomorphism in dimension $2^{k} m$ and thus ker $i=\operatorname{ker} \delta^{\prime}$.

We are done if we show $\delta^{\prime}\left(y^{2}\right)=0$. Now $\pi \delta^{\prime}\left(y^{2}\right)=\delta_{(1)}\left(y^{2}\right)=0$ since $\delta_{(1)}$ is a derivation. But under our hypothesis $\pi$ is a monomorphism on $\left(H^{2 k+1}\left(X, Z_{2}\right)\right)_{2}$ and $\delta^{\prime}\left(y^{2}\right)$ is surely in this subgroup.

We now discuss the possibility of carrying out a similar construction with regard to the homology product. We first consider the case where the homology ring is commutative:

2.12. Definition. Let $X$ be an $H$-space with a commutative homology ring. Let $\pi: Z_{4} \rightarrow Z_{2}$. We define $P_{2}^{\prime}(\operatorname{Im} \pi) \subseteq H_{2 m}\left(X, Z_{2}\right) \rightarrow H_{4 m}\left(X, Z_{4}\right)$ by $P_{2}^{\prime}(x)=$ $\left(\pi^{-1}(x)\right)^{2}$.

The following lemmas and theorems proceed exactly as before.

2.13. LEMMA. $P_{2}^{\prime}$ is well defined and

(a) $P_{2}^{\prime}(x+y)=P_{2}^{\prime}(x)+P_{2}^{\prime}(y)+2_{*} x \cdot y$,

(b) $\pi P_{2}^{\prime}(x)=x^{2}$.

2.14. TheOREM. Let $\hat{P}_{2}^{\prime}:(\operatorname{Im} \pi) \rightarrow H_{4 m}\left(X, C_{2}\right)$ be defined by $\hat{P}_{2}^{\prime}(x)=$ $\widehat{P_{2}^{\prime}(x)}$. Then $\hat{P}_{2}^{\prime}$ induces operations, $\hat{P}_{2}^{\prime r}: E_{2 m}^{r} \rightarrow D_{4 m}^{r}, r>1$, and

(a) $\hat{P}_{2}^{\prime r}(x+y)=\hat{P}_{2}^{\prime r}(x)+\hat{P}_{2}^{\prime r}(y)+2_{*}(x \cdot y)$,

(b) $c \hat{P}_{2}^{\prime r}(x)=\widehat{x^{2}}$. 
2.15. THEOREM. There is a well-defined pairing $\varphi^{\prime}: \operatorname{ker} q_{2 m}^{\prime r} \times \operatorname{Im} \delta_{(r)}^{4 m-1}$ $\rightarrow Z_{2}, r>1$. $\ni$.

(a) $\varphi^{\prime}\left(x, y,+y_{2}\right)=\varphi^{\prime}(x, y)+\varphi^{\prime}\left(x, y_{2}\right)$,

(b) $\varphi^{\prime}\left(x+x_{2}, y\right)=\varphi^{\prime}(x, y)+\varphi^{\prime}\left(x_{2}, y\right)+\left\langle x_{1} \cdot x_{2}, y\right\rangle$,

(c) if $f: X \rightarrow X^{\prime}$ then $\varphi^{\prime}\left(f_{*}(x), y^{\prime}\right)=\varphi^{\prime}\left(x, f^{*}\left(y^{\prime}\right)\right)$.

2.16. THEOREM. $r>1$. Let $X$ be an H-space with commutative homology ring. Let $0 \neq \bar{x} \in \operatorname{Im} \delta_{(r)}^{2 n-1}$ be primitive. Let $x$ be its dual and $X \in \operatorname{ker} q_{r}^{\prime 2 m}$. Then $\varphi^{\prime}\left(x, \bar{x}^{2}\right) \neq 0$; hence $\bar{x}^{2} \neq 0$.

2.17. Remarks. Finally, in the homotopy commutative case one may actually construct operations that behave as do the divided power operations and induce $\hat{P}_{2}^{\prime}$. Hence in this case one may drop the restriction $r>1$.

3. On the construction of Bockstein towers. In this section we discuss the problem of enumerating the Bockstein towers with respect to a given $R$-module $A$. In particular, we ask when a tower can be constructed over a given sequence $0 \rightarrow$ $A \stackrel{\alpha_{1}}{\rightarrow} A_{1} \stackrel{\beta_{1}}{\rightarrow} A \rightarrow 0$ representing an element $a \in \operatorname{Ext}_{R}^{1}(A, A)$. The approach taken is to attempt to extend the tower one row at a time. At each stage a primary obstruction appears which must vanish in order to extend further. However, even if the primary obstruction does not vanish, there is presented a secondary obstruction which still may vanish. The vanishing of the secondary obstruction allows one to modify the top row of the tower (leaving the rest fixed) so as to be sure the new primary obstruction vanishes.

While in general higher obstructions still may be required for the examples considered below, these two levels of obstruction are shown to be sufficient to enumerate the towers over the given $A$.

3.1. Setring. We restrict attention to the category of $R$-modules. At one point below it is necessary to assume $A$ is a simple $R$-module of finite cardinality. We will make this assumption only at the place it is needed. Of course, in the usual applications of the Bockstein spectral sequence this condition is present.

The basic diagram for this section is

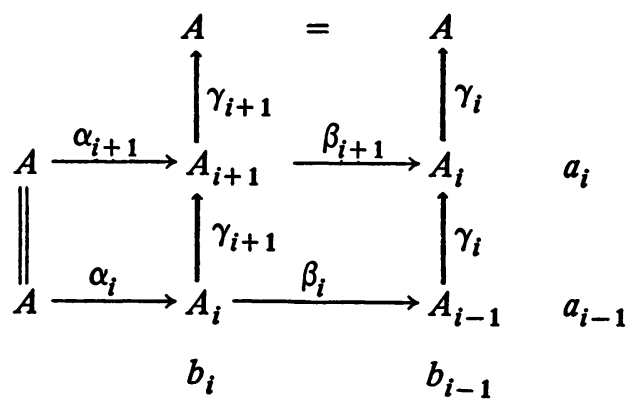


where $a_{j} \in \operatorname{Ext}_{R}^{1}\left(A_{j}, A\right), b_{j} \in \operatorname{Ext}_{R}^{1}\left(A, A_{j}\right), j=i-1$, and $i$ represents the appropriate row or column. We will let $\delta_{b_{j}}: \operatorname{Ext}_{R}^{1}\left(A_{j}, A\right) \rightarrow \operatorname{Ext}_{R}^{2}(A, A)$ stand for the boundary homomorphism in the long exact sequence associated with the appropriate column. Finally, it is assumed that this is the top row of a partial tower over a given sequence $A \stackrel{\alpha_{2}}{\longrightarrow} A_{1} \stackrel{\beta_{1}}{\longrightarrow} A$.

The following is the basic observation.

3.3. THEOREM. Suppose $\delta_{b_{i}}\left(a_{i}\right)=0$ then we may extend the tower one more row. That is, find

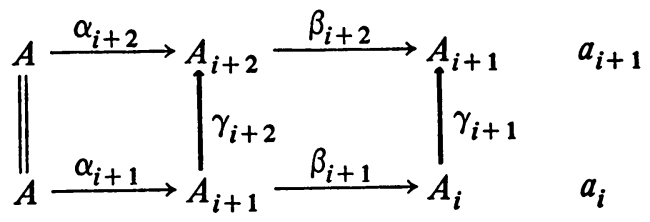

Proof. $\delta_{b_{i}}\left(a_{i}\right)=0$ implies that there exists $a_{i+1} \in \operatorname{Ext}_{R}^{1}\left(A_{i+1}, A\right)$ with $\gamma_{i+1}^{*}\left(a_{i+1}\right)=a_{i}$.

The following observations require hypotheses that are too strong to be useful in general.

3.4. Corollary. Suppose $\operatorname{Ext}_{R}^{2}(A, A)=0$; then there exists a tower over each element in $\operatorname{Ext}_{R}^{1}(A, A)$.

3.5. THEOREM. Suppose $\delta_{b_{i}}\left(a_{i}\right)=0$ and $\gamma_{i}^{*}$ is a monomorphism; then $a_{i+1}$ is unique.

Proof. Studying the long sequences associated with the rows

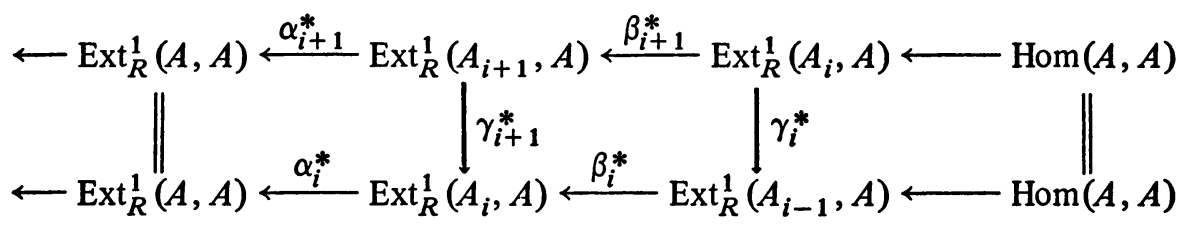

the result follows from the five lemma.

3.6. Corollary. Suppose $\operatorname{Ext}_{R}^{2}(A, A)=0$ and $\alpha_{1}^{*}=\gamma_{1}^{*}$ is a monomorphism, then there is a unique tower over each element of $\operatorname{Ext}_{R}^{1}(A, A)$.

Proof. By induction, $\gamma_{i}^{*}$ a monomorphism implies $\gamma_{i+1}^{*}$ is a monomorphism. This fact and 3.4 give 3.6.

Before developing the obstruction theory of Bockstein towers further we give a series of examples which exhibit the various phenomena to be delineated. In all cases the " $A$ " in question will be taken to be $Z_{2}$. We do not give details of the various computations since in all cases they are straightforward. 
3.7. Examples. (a) Z-modules. Here $\operatorname{Ext}_{Z}^{1}\left(Z_{2}, Z_{2}\right)=Z_{2}, \operatorname{Ext}_{Z}^{2}\left(Z_{2}, Z_{2}\right)$ $=0$. Moreover, $\alpha_{1}^{*}$ is a monomorphism. Thus 3.6 applies.

(b) $Z[Z]$-modules. $\operatorname{Ext}_{Z[Z]}^{1}\left(Z_{2}, Z_{2}\right)=Z_{2} \oplus Z_{2}$, $\operatorname{Ext}_{Z[Z]}^{2}\left(Z_{2}, Z_{2}\right)=Z_{2}$. By direct computation one can show $\delta_{b_{i}} a_{i}=0$ for diagrams of type (3.2). Of course uniqueness is lost.

(c) $Z\left[Z_{2}\right]$-modules. $\operatorname{Ext}_{Z\left[Z_{2}\right]}^{1}\left(Z_{2}, Z_{2}\right)=Z_{2} \oplus Z_{2}, \operatorname{Ext}_{Z\left[Z_{2}\right]}^{2}\left(Z_{2}, Z_{2}\right)=$ $Z_{2} \oplus Z_{2}$ (generated by, say, $u_{1}$ and $u_{2}$ ).

Using example (b) and the fact that the change of rings homomorphism $\operatorname{Ext}_{Z\left[Z_{2}\right]}^{2}\left(Z_{2}, Z_{2}\right) \rightarrow \operatorname{Ext}_{Z[Z]}^{2}\left(Z_{2}, Z_{2}\right)$ is the "folding map", one sees that $\delta_{b_{i}}\left(a_{i}\right)$ $=0$ or $u_{1}+u_{2}$. On the other hand, one may choose generators $v_{1}$ and $v_{2}$ of $\operatorname{Ext}_{Z\left[Z_{2}\right]}^{1}\left(Z_{2}, Z_{2}\right)$,

$$
\delta_{v_{j}}\left(v_{i}\right)= \begin{cases}0, & i=j, \\ u_{j}, & i \neq j .\end{cases}
$$

The following example shows that $\delta_{b_{i}}\left(a_{i}\right)$ is not always zero.

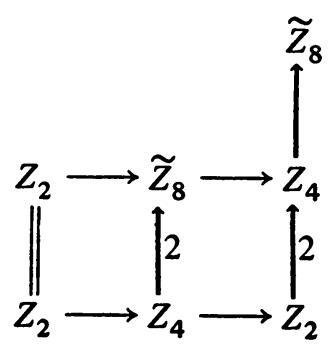

where $\widetilde{Z}_{8}$ is $Z_{8}$ with the $Z_{2}$-action $\varphi(x)=5 x$. Note that $Z_{16}$ does not admit a $Z_{2}$. action which lifts $\varphi$. Note also that one can modify the action $\varphi$ so that there is a lifting (replace $\varphi$ by the trivial action).

(d) $Z_{8}$-modules. $\operatorname{Ext}_{Z_{8}}^{1}\left(Z_{2}, Z_{2}\right)=Z_{2}, \operatorname{Ext}_{Z_{8}}^{2}\left(Z_{2}, Z_{2}\right)=Z_{2}$.

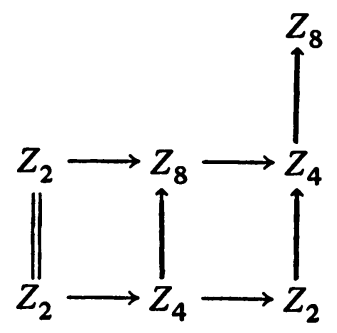

does not "lift" as $Z_{\mathbf{8}}$-modules nor can one modify the top row so that it does lift.

We now need a technical lemma which is, in fact, about the bilinear pairing in Ext [6]. 
3.8. Lemma. Let $b_{1}, b_{2} \in \operatorname{Ext}_{R}^{n}(A, D)$. Then $\delta_{b_{1}+b_{2}}(a)=\delta_{b_{1}}(a)+$ $\delta_{b_{2}}(a)$ in $\operatorname{Ext}_{R}^{n+1}(C, D)$.

Consider the diagram defining addition in $\operatorname{Ext}_{R}^{1}(C, A)[6]$ :

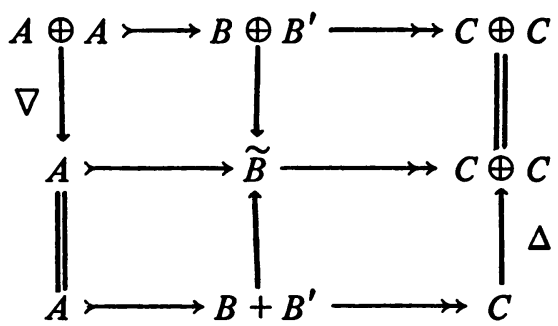

Now $\delta_{b+b^{\prime}}(a)=\Delta^{*}\left(\delta_{b} \oplus \delta_{b^{\prime}}\right) \nabla^{*}(a)$ and $\nabla^{*}(x)=x \oplus x$ and $\Delta^{*}(x \oplus y)=x+y$.

The following lemmas show the relation between the elements $a_{i}$ and $b_{i}$ in (3.2). The lemmas are stated in terms of the following "Ext" diagram with exact rows and columns.
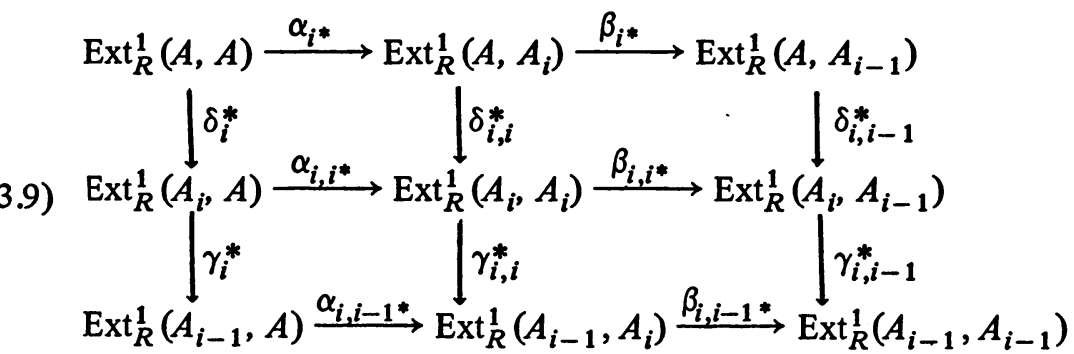

3.10. LEMMA. $\delta_{i, i}^{*}\left(b_{i}\right)=\alpha_{i, i^{*}}\left(a_{i}\right)$.

Proof. In the following diagram we are given all but the map $\zeta$.

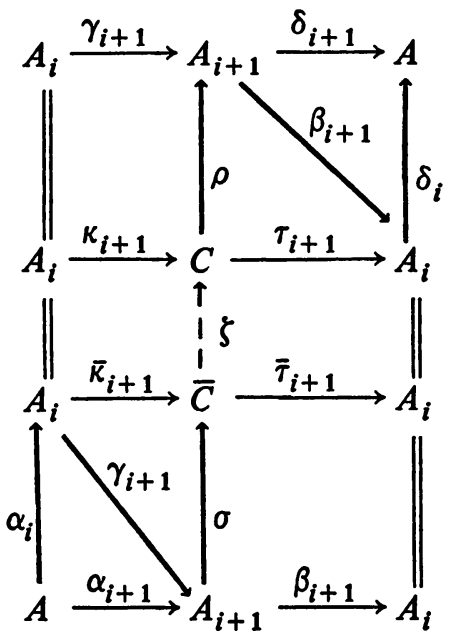


where the second and third rows represent $\delta_{i, i}^{*}\left(b_{i}\right)$ and $\alpha_{i, i^{*}}\left(a_{i}\right)$.

We now define the map $\zeta: \bar{C} \rightarrow C$. First remember that $\bar{C}$ is the push out of

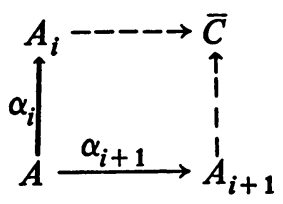

and $C$ is the pull back of

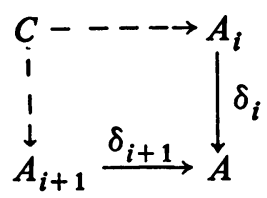

Define $\zeta\left(x_{i}, x_{i+1}\right)$ by $\zeta\left(x_{i}, 0\right)=\left(\gamma_{i+1} x_{i}, 0\right), \zeta\left(0, x_{i+1}\right)=\left(x_{i+1}, \beta_{i+1} x_{i+1}\right)$.

We first check that this map is well defined, in particular, that

$$
\zeta\left(\alpha_{i} x,\left(-\alpha_{i+1} x\right)\right)=\left(\alpha_{i+1} \alpha_{i} x-\alpha_{i+1} x,-\beta_{i+1} \alpha_{i+1} x\right)=(0,0) .
$$

Similarly one shows $\kappa_{i+1}=\zeta \bar{\kappa}_{i+1}, \tau_{i+1} \zeta=\tau_{i+1}$; hence $\zeta$ is an isomorphism by the five lemma. We note $\rho \zeta \sigma=$ ident. This can be seen by direct computation.

We now prove a partial converse under the assumption $A$ is a simple $R$-module of finite cardinality.

We begin by noting that the modules appearing in any nontrivial tower are all essential extensions of $A$.

3.11. Lemma. Let $A$ be simple and $A \stackrel{\alpha_{1}}{\longrightarrow} A_{1} \stackrel{\beta_{1}}{\longrightarrow} A$ nontrivial. Then $A \stackrel{\alpha_{i}}{\longrightarrow} A_{i}$ is essential.

Proof. Consider the diagram

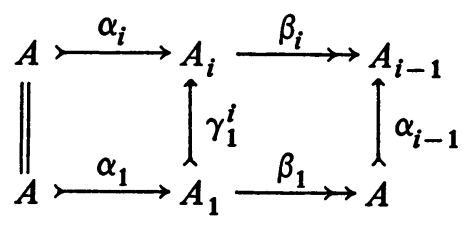

Defining $\gamma_{1}^{1}$ and $\alpha_{0}$ to be the identity we may assume $\alpha_{i-1}: A \rightarrow A_{i-1}$ is essential. Now if $\alpha_{i} A \rightarrow A_{i}$ is not essential let $M \subseteq A_{i}, M \cap \alpha_{i}(A)=0, M \neq 0$. $M \cap \alpha_{i}(A)=0$ implies $\beta_{i} / M$ is a monomorphism. $\alpha_{i-1}$ essential implies $\alpha_{i-1}(A)$ $\subseteq \beta_{i}(M)$; hence there is a map $\tau: \alpha_{i-1}(A) \rightarrow A_{i} . \ni . \beta_{i} \tau=$ ident. But $\beta_{i}^{-1} \alpha_{i-1}(A)$ $=\gamma_{1}^{i}\left(A_{1}\right)$ so $\tau$ is a splitting of the bottom row contradicting the hypothesis.

We are now in a position to prove a partial converse to 3.10 . 
3.12. THEOREM. Let $A$ be simple and of finite cardinality. Let $\bar{a} \in$ $\operatorname{Ext}_{R}^{1}\left(A_{i}, A\right), \bar{b} \in \operatorname{Ext}_{R}^{1}\left(A, A_{i}\right)$ be $\ni$; (a) $\gamma^{*}(\bar{a})=a_{i-1}$; (b) $\beta_{i^{*}}(\bar{b})=b_{i-1}$; and (c) $\alpha_{i, i}(\bar{a})=\delta_{i, i}^{*}(\bar{b})$. Then there exists a diagram

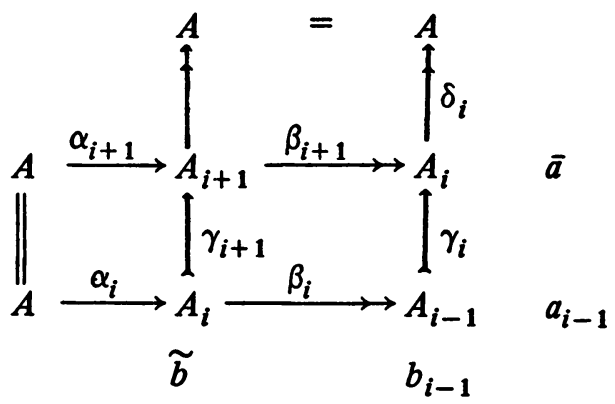

with $\delta_{\bar{b}}=\delta_{\widetilde{b}} f^{*}$ where $f: A_{i} \rightarrow A_{i}$ is an automorphism.

Proof. Consider the following diagram associated with the above data.

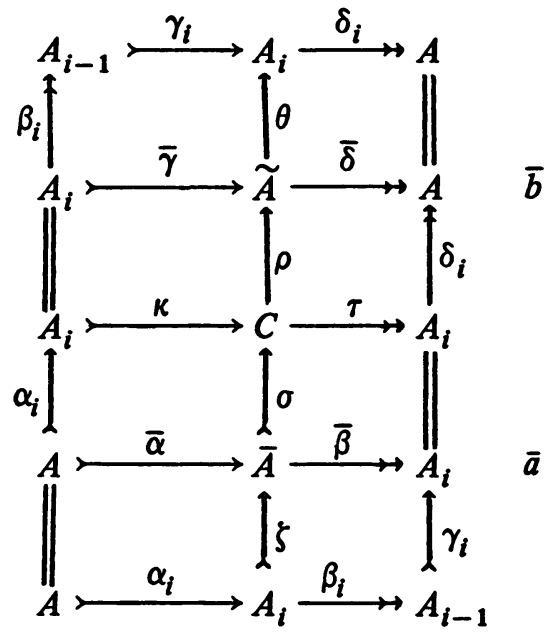

We wish to show $\rho \sigma$ is an isomorphism. To do this we first observe $\operatorname{card}(\widetilde{A})$ $=\operatorname{card}(\bar{A})$ since they are both extensions of modules that are pairwise of the same cardinality. Therefore, to show $\rho \sigma$ is an isomorphism it is sufficient to show it is $1-1$.

Suppose $\rho \sigma(x)=0$. Then $\delta \rho \sigma(x)=0$; hence by commutativity $\delta_{i} \bar{\beta}(x)=0$. Hence $\bar{\beta}(x) \in \operatorname{ker}\left(\delta_{i}\right)=$ image $\gamma_{i}$. This implies that there exists $y \in A_{i}$ such that $\zeta(\mathrm{y})=x$. On the other hand $\alpha_{i}(A) \cap \operatorname{ker} \delta \sigma \zeta=\operatorname{ker} \bar{\gamma} \alpha_{i}=0$ but, by 3.10, $\alpha_{i}$ is essential so ker $\rho \sigma \xi=0=>y=0=>x=0$. 
We claim that the following diagram satisfies the requirements of our theorem.

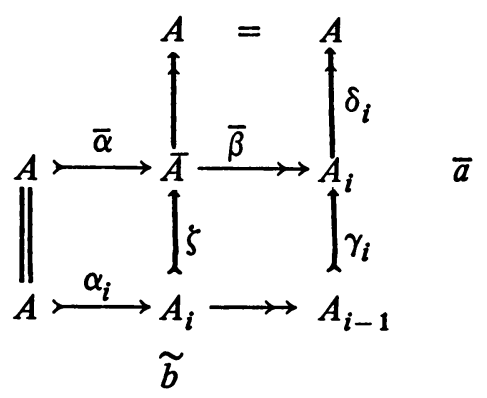

To see this one considers

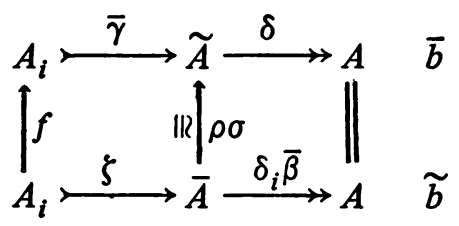

By commutativity $\rho \sigma \zeta$ factors through $\bar{\gamma}$ which, of necessity, must be an automorphism of $A_{i}$.

Applications of 3.12 unfortunately require knowledge of the automorphism $f$. We will see that in the case we study this is available. Moreover, we will mention an alternate version of 3.12 which identifies a situation where we are sure $f$ is the identity.

3.13. Continuation of Example 3.7(c). We study the towers over $Z_{2}$ as a $Z\left[Z_{2}\right]$-module. Firstly, there are three nonzero elements of $\operatorname{Ext}_{Z\left[Z_{2}\right]}^{1}\left(Z_{2}, Z_{2}\right), u_{1}$ representing $Z_{2} \rightarrow Z_{4} \rightarrow Z_{2}, u_{2}$ representing $Z_{2} \rightarrow Z_{4} \rightarrow Z_{2}$ and $u_{1}+u_{2}$ representing $Z_{2} \rightarrow Z_{2} \oplus Z_{2} \rightarrow Z_{2}$ with the nontrivial action. As indicated in 3.7(c) $\delta_{u_{1}} u_{1}=\delta_{u_{2}} u_{2}=0$ but $\delta_{u_{1}+u_{2}}\left(u_{1}+u_{2}\right)=v_{1}+v_{2}$ hence all towers over $Z_{2}$ are over $u_{1}$ or $u_{2}$.

It is a simple observation that any tower over either considered as a tower of $Z$-modules is just $\left(Z_{2} i, 2\right)(1.6(\mathrm{a}))$; hence $A_{i}=Z_{2} i+1$ with some $Z_{2}$ action. Again it is easy to verify that for any such module and automorphism $f: A_{i} \rightarrow$ $A_{i}, f^{*}=$ ident: $\operatorname{Ext}_{Z\left[Z_{2}\right]}^{1}\left(A_{i}, Z_{2}\right) \rightarrow \operatorname{Ext}_{Z\left[Z_{2}\right]}^{1}\left(A_{i}, Z_{2}\right)$. Also one sees $\operatorname{Ext}_{Z\left[Z_{2}\right]}^{1}\left(A_{i}, Z_{2}\right)=Z_{2} \oplus Z_{2}$ and $\alpha_{i, i}, \delta_{i, i *}^{*}$ of (3.9) are both monomorphisms.

Suppose, we have constructed a tower as in (3.2) and $\delta_{b_{i}} a_{i} \neq 0$, by 3.7(b) and (c), we have $\delta_{b_{i}} a_{i}=v_{1}+v_{2}$. Suppose $\alpha_{i}^{*} a_{i}=u_{j}=\delta_{i *} b_{i}, j=1$ or 2 (say $j=1)$. Consider the pair $\delta_{i}^{*} u_{2}+a_{i}, \alpha_{i} u_{2}+b_{i}$ by 3.12 and the fact $f^{*}=$ ident. We may modify the tower so that the obstruction to lifting is 


$$
\begin{aligned}
& \delta_{\left(\alpha_{i} * u_{2}+b_{i}\right)}\left(\delta_{i}^{*} u_{2}+a_{i}\right) \\
& =\delta_{\alpha_{i^{*} u_{2}}} \delta_{i}^{*} u_{2}+\delta_{\alpha_{i^{*} u_{2}} a_{i}}+\delta_{b_{i}} \delta_{i}^{*} u_{2}+\delta_{b_{i}} a_{i} \\
& =\delta_{u_{2}}\left(\alpha_{i}^{*} \delta_{i}^{*} u_{2}\right)+\delta_{u_{2}} \alpha_{i}^{*} a_{i}+\delta_{\delta * i b_{i}} u_{2}+\delta_{b_{i}} a_{i} \\
& =0+\delta_{u_{2}} u_{1}+\delta_{u_{1}} u_{2}+\delta_{b_{i}} a_{i} \\
& =0+v_{2}+v_{1}+v_{1}+v_{2} \\
& =0 \text {. }
\end{aligned}
$$

Moreover, again one may quickly check that this pair is the only pair over $a_{i-1}, b_{i-1}$ that lifts. Hence there is a unique tower over $u_{1}$ namely $\left(Z_{2} i, 2\right)$. Similarly there is a unique tower over $u_{2}$ namely $\left(\hat{Z}_{2} i, 2\right)$. Hence these generate the only Bockstein spectral sequences over $Z_{2}$ as a $Z\left[Z_{2}\right]$-module.

We finish by making one further observation suggested by the above which we leave unproved.

3.14. Theorem. In the setting of (3.2) and (3.9) let $a^{\prime} \in \operatorname{Ext}_{R}^{1}(A, A)$. Then there is a diagram of type (3.2) with middle row and column $a_{i}+\delta_{i}^{*} a^{\prime}, b_{i}+$ $\alpha_{i *} a^{\prime}$.

The proof consists of merely checking that the map $f$ of 3.12 is in this case actually the identity.

\section{BIBLIOGRAPHY}

1. W. Browder, Torsion in H-spaces, Ann. of Math. (2) 74 (1961), 24-51. MR 23 \#A2201.

2. - Homotopy commutative H-spaces, Ann. of Math. (2) 75 (1962), 283-311. MR 27 \#765.

3. W. Browder and I. Namioka, H-spaces with commutative homology rings, Ann. of Math. (2) 75 (1962), 449-451. MR 26 \#5579.

4. J. L. Dupont and G. Lusztig, On manifolds satisfying $w_{1}^{2}=0$, Topology 10 (1971), 81-92. MR $42 \# 8508$.

5. B. Eckmann and P. J. Hilton, Exact couples in an abelian category, J. Algebra 3 (1966), 38-87. MR 33 \#164.

6. S. Mac Lane, Homology, Die Grundlehren der math. Wissenschaften, Band 114, Academic Press, New York; Springer-Verlag, Berlin, 1963. MR 28 \#122.

7. E. Thomas, The generalized Pontriagin cohomology operations and rings with divided powers, Mem. Amer. Math. Soc. no. 27 (1957). MR 20 \#5474.

DEPARTMENT OF MATHEMATICS, UNIVERSITY OF MISSOURI, ST. LOUIS, MISSOURI 63121 